\title{
Comparison between transumbilical and transvaginal morcellation of a large uterus during single-port-access total laparoscopic hysterectomy
}

\author{
Seung Ju Oh, MD¹, Seo Young Lee, MD¹, Woo Yong Kim, MD², Jieun Kang, MD¹, Kyoung-Hee Han, MD', \\ San-Hui Lee, MD ${ }^{1,3}$, Ji Sun Park, MD'1 \\ Department of Obstetrics and Gynecology, ${ }^{1}$ Yonsei University Wonju College of Medicine, Wonju; ${ }^{2}$ Kangbuk Samsung Hospital, Sungkyunkwan \\ University School of Medicine, Seoul; ${ }^{3}$ National Health Insurance Service Ilsan Hospital, Goyang, Korea
}

\section{Objective}

To compare the perioperative outcomes of transumbilical morcellation (TUM) and transvaginal morcellation (TVM) of a large uterus ( $\geq 500 \mathrm{~g}$ ) during single-port-access total laparoscopic hysterectomy (SPA-TLH).

\section{Methods}

A total of 57 patients who underwent SPA-TLH for a large uterine myoma and/or adenomyosis (uterine weight $\geq 500 \mathrm{~g}$ ) between March 2013 and July 2017 were included. For specimen retrieval, TUM was performed for 30 patients and TVM for 27 patients.

\section{Results}

Perioperative outcomes, including total operative time, tissue extraction time, extension of skin incision length, estimated volume of blood loss, changes in postoperative hemoglobin level, length of postoperative hospital stay, postoperative pain, and uterine weight, were compared between the 2 groups. No significant differences were observed in the baseline characteristics except for a history of cesarean section (TUM vs. TVM: $83.3 \%$ vs. $14.8 \%, P=0.002$ ) and history of vaginal delivery (TUM vs. TVM: $6.7 \%$ vs. $88.8 \%, P=0.001$ ). The total operative time, tissue extraction time, extension of skin incision length, estimated volume of blood loss, changes in postoperative hemoglobin level, length of postoperative hospital stay, and postoperative pain did not significantly differ between the two groups. The uterine weight was significantly higher for patients who underwent TUM than for those who had TVM (median [range]: $735 \mathrm{~g}$ [520-1,380 g] vs. $622 \mathrm{~g}$ [514-975 g]; $P=0.042$ ).

\section{Conclusion}

TUM during SPA-TLH is a feasible technique for extracting large uteri weighing $\geq 500 \mathrm{~g}$. This procedure is suitable for patients without a history of vaginal delivery or a narrow vaginal cavity.

Keyword: Adenomyosis; Hysterectomy; Laparoscopy; Minimally invasive surgical procedures; Myoma

\section{Introduction}

Since laparoscopic hysterectomy was first performed [1], a series of studies have shown the superiority of laparoscopic hysterectomy over open hysterectomy in terms of better view of the surgical field, low volume of blood loss, faster recovery, and decreased wound complications [2]. Furthermore, to maximize the advantages of laparoscopic surgery and reduce the incision size and number of trocars [3], single-port laparoscopy was carried out.
Received: 2019.07.16. Revised: 2019.10.28. Accepted: 2019.11.07. Corresponding author: Ji Sun Park, MD

Department of Obstetrics and Gynecology, Yonsei University Wonju College of Medicine, 20 Ilsan-ro, Wonju 26426, Korea E-mail: j880322@gmail.com

https://orcid.org/0000-0001-9522-0046

Articles published in Obstet Gynecol Sci are open-access, distributed under the terms of the Creative Commons Attribution Non-Commercial License (http://creativecommons. org/licenses/by-nc/3.0/) which permits unrestricted non-commercial use, distribution, and reproduction in any medium, provided the original work is properly cited.

Copyright $\odot 2020$ Korean Society of Obstetrics and Gynecology 


\section{Obstetrics \& Gynecology Science}

Vol. 63, No. 3, 2020

After single-port-access total laparoscopic hysterectomy (SPA-TLH) was first performed in 1991 [4], the universalization of single-port laparoscopy has been slow. However, single-port laparoscopy has been more commonly used in the gynecologic field after the latest developments in laparoscopic instrumentation and the accumulation of surgical experience [5].

In SPA-TLH, specimen retrieval is one of the most critical factors affecting operative time, and the length of the procedure is positively correlated with uterine weight [6]. Efforts have been made to reduce specimen retrieval time in SPATLH, which include performing procedures, such as intraabdominal morcellation, transvaginal morcellation (TVM), and combined laparoscopic morcellation and TVM $[7,8]$. Specimen retrieval during umbilicus and manual morcellation of a large specimen in a gynecological laparoscopic operation is considered feasible and safe [9]. Furthermore, in some cases, the transumbilical morcellation (TUM) of a large uterus had a shorter operative time [10].

The current study aimed to compare the surgical outcomes between TUM and TVM for retrieving a large uterus ( $\geq 500 \mathrm{~g}$ ) during SPA-TLH.

\section{Materials and methods}

This retrospective study included all patients who underwent SPA-TLH between March 2013 and July 2017 at two institutions (National Health Insurance Service Ilsan Hospital, Goyang; Wonju Severance Christian Hospital, Wonju). During the study period, 386 patients underwent SPA-TLH for benign diseases, and a single surgeon performed all procedures. Among the patients, those with uterine weights over $500 \mathrm{~g}$ based on the pathology report were selected. Thus, 57 patients were included in this study. The data of 30 and 27 patients who underwent SPA-TLH with TUM and TVM, respectively, for tissue extraction were compared. All patients underwent transvaginal and/or transabdominal ultrasonography within 30 days before surgery, during which the size of the uterus was assessed and recorded. If the diagnosis and evaluation of the uterine mass via ultrasonographic examination were not sufficient, additional abdominopelvic computed tomography scan or magnetic resonance imaging was performed.

The following patients met the inclusion criteria of this study: women with a benign gynecological disease, such as uterine myoma and adenomyosis, who underwent total laparoscopic hysterectomy, had a uterus weighing $\geq 500 \mathrm{~g}$ were classified as American Society of Anesthesiologists class I and II, and were aged under 60 years.

Due to a high risk of undergoing complex surgical procedures, such as adhesiolysis or resection of severe endometriosis, patients with more than 4 previous abdominopelvic surgeries via laparotomy or those with clinical evidence of deep infiltrating endometriosis were excluded from the study. Moreover, patients diagnosed with malignancy via pathological examinations were excluded.

TUM or TVM was performed based on manual pelvic examination. Patients with a narrow vagina ( $<2$ finger breadths) were subjected to specimen retrieval via TUM [11].

A specific type was designed to retrospectively collect data about the characteristics of the patients, intraoperative details, surgical outcomes, and perioperative complications. The total operative time was defined as the time from skin incision to skin closure. The tissue extraction time was defined as the time spent on tissue extraction. The data were collected from the electronic medical records. In the anesthesiology unit, the estimated volume of blood loss was calculated as the difference between the total amount of suction and irrigation plus the difference between total gauze weight before and after surgery. To determine the extent of skin incision before and after surgery, the length of the skin incision was measured before inserting the single-port channel system and after skin closure (Fig. 1).

Changes in hemoglobin level was defined as the difference
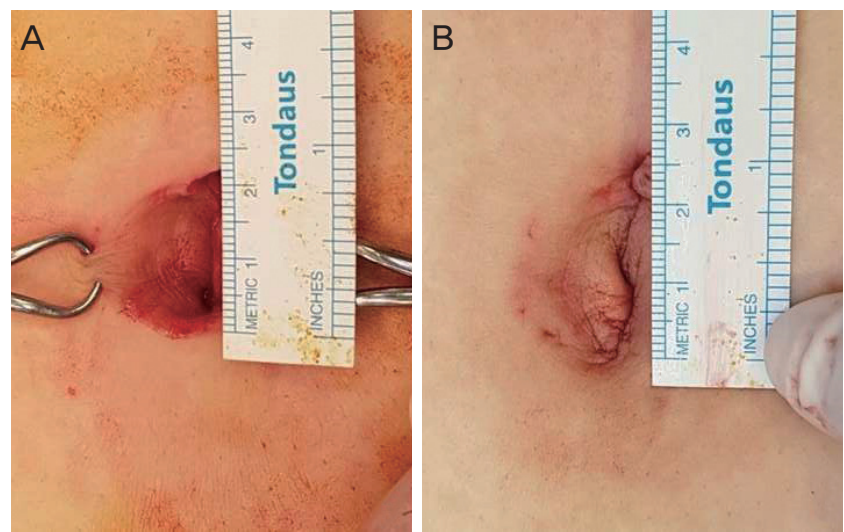

Fig. 1. Umbilicus wound (A) before starting laparoscopy, (B) after the end of operation. 


\section{Obstetrics \& Gynecology Science}

Seung Ju Oh, et al. Transumbilical \& transvaginal morcellation

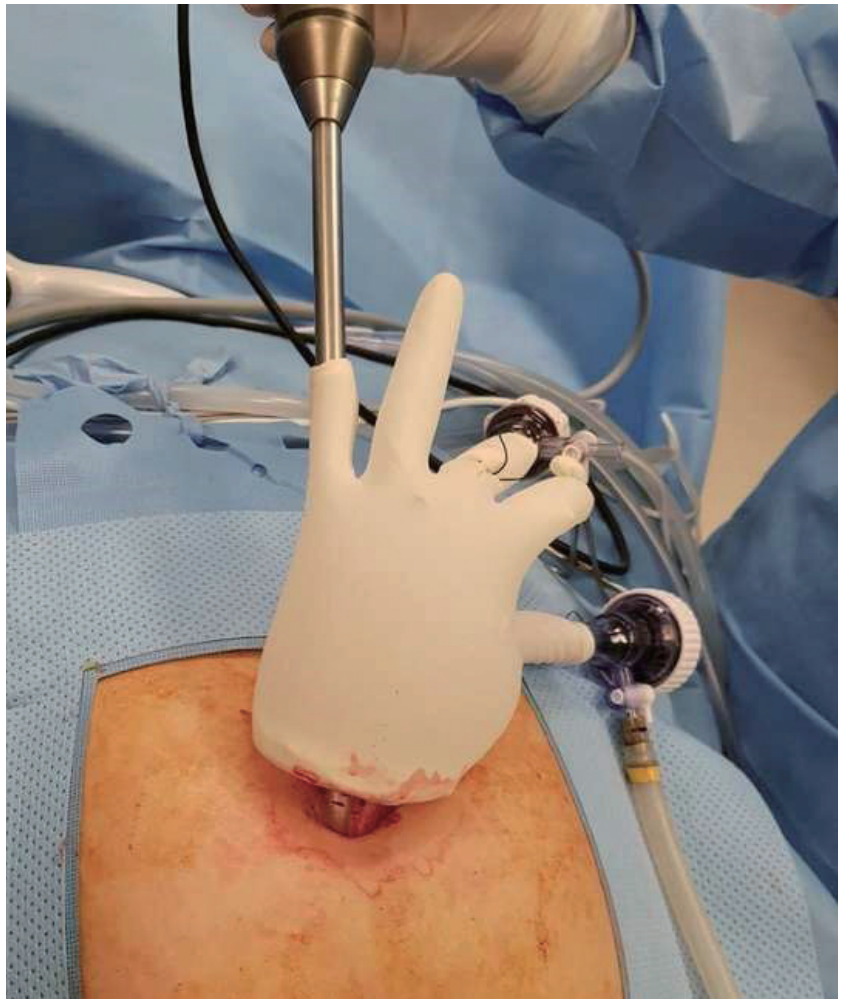

Fig. 2. Single-port channel system. between preoperative hemoglobin level and hemoglobin level on postoperative day 1. Patients were discharged from the hospital after the return of bowel activity and a successful ambulation and if fever was not observed after surgery and narcotic analgesics were no longer required. Length of hospital stay was defined as the time from the operative day to the day of discharge. Several assessors who were not associated with the investigators performed postoperative pain assessments with a visual analog scale 12, 24, and 48 hours after surgery. The scale was presented as a $10-\mathrm{cm}$ line with the verbal descriptors "no pain" and "pain as bad as it could be" as the final outcome [12]. All intraoperative and postoperative complications arising within 30 days of surgery were recorded. All patients were scheduled for follow-up examinations at 1 week and 1 month after surgery.

\section{Surgical technique}

A single surgeon performed all surgeries at the two institutions. A single-port channel system with surgical glove and Alexis wound protectors/retractors (Applied Medical, Rancho Santa Margarita, CA, USA) was inserted through the umbili-
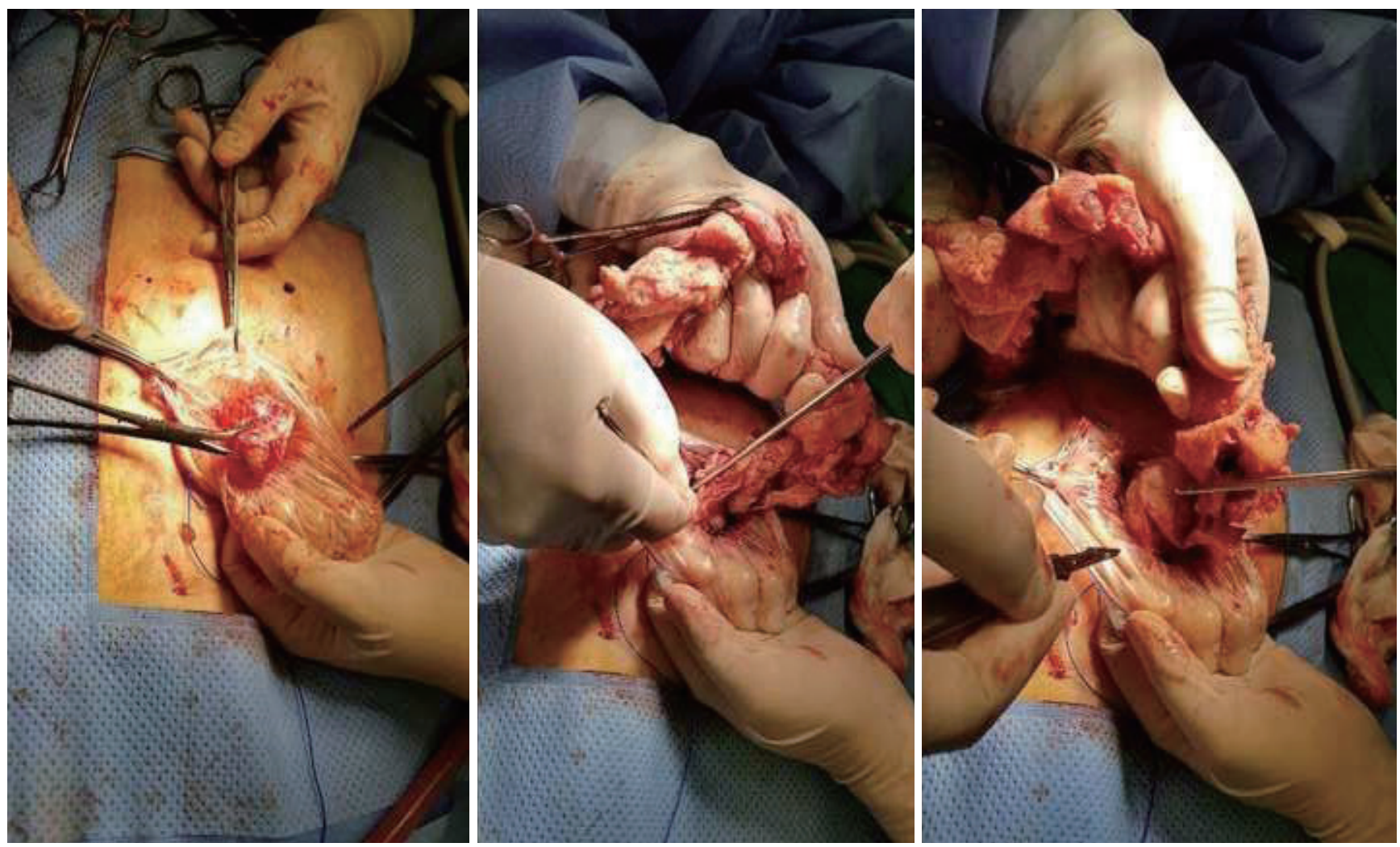

Fig. 3. Tissue extraction through umbilicus. 


\section{Obstetrics \& Gynecology Science}

Vol. 63, No. 3, 2020

cus (Fig. 2). Hysterectomy was performed with conventional straight laparoscopic instruments and advanced bipolar instruments, such as LigaSure ${ }^{\mathrm{TM}}$ (Covidien, Dublin, Ireland) and THUNDERBEAT (Olympus Medical, Tokyo, Japan). In both TVM and TUM cases, the uterus was placed in an endopouch (Lap Bag ${ }^{\mathrm{TM}}$ metal type; Meditech Infrared, Seoul, Korea) before morcellation.

In TVM cases, tissue extraction was performed before vault closure. After placing the right-angle retractors at the 12 and 6 o'clock positions to retract the anterior and posterior vaginal walls and to protect the urethra, urinary bladder, and rectum, the uterine cervix was grasped with towel clips or Lahey clamps for pulling. Starting from the cervix, the uterus was circumferentially incised with scalpels or with Mayo scissors.

In TUM cases, the edge of the endo-pouch was elevated via an umbilical incision, and the specimen was visualized. The uterus was held and pulled with towel clips or Lahey clamps while it was circumferentially incised with a scalpel. The specimen was extracted in small fragments (Fig. 3).

The vaginal cuff was closed, and the peritoneum, fascia, and subcutaneous tissue were then estimated and closed layer by layer using 2-0 Polysorb sutures (Covidien). Reinforced skin closures (Steri-Strip TM; 3M Health Care, St. Paul, MN, USA) were used to close the incision.

\section{Statistical analysis}

All statistical analyses were performed using the Statistical Package for the Social Sciences software version 23.0 (SPSS Inc., Chicago, IL, USA). Data were presented as means \pm standard deviations or medians (ranges) for continuous variables and frequencies (percentages) for categorical variables. The baseline clinical characteristics and study outcomes were compared between the two groups using the $\chi^{2}$ test or Fisher's exact tests for categorical variables and the student's $t$-test or Mann-Whitney $U$ test for continuous variables accordingly. A $P$-value $<0.05$ was considered statistically significant.

Table 1. Baseline characteristics $(n=57)$

\begin{tabular}{|c|c|c|c|}
\hline & TUM $(n=30)$ & TVM $(n=27)$ & $P$-value \\
\hline Age (yr) & $45.2 \pm 5.8$ & $46.8 \pm 7.2$ & 0.892 \\
\hline Body mass index $\left(\mathrm{kg} / \mathrm{m}^{2}\right)$ & $24.2 \pm 4.2$ & $24.9 \pm 4.1$ & 0.341 \\
\hline Parity & & & $>0.986$ \\
\hline Nulliparous & $2(6.7)$ & $1(3.7)$ & \\
\hline Parous & $28(93.3)$ & $26(96.3)$ & \\
\hline History of cesarean section & $25(83.3)$ & $4(14.8)$ & 0.002 \\
\hline 1 & 11 & 1 & \\
\hline 2 & 13 & 3 & \\
\hline$\geq 3$ & 1 & 0 & \\
\hline History of vaginal delivery & $2(6.7)$ & $24(88.8)$ & 0.001 \\
\hline \multicolumn{4}{|l|}{ Hysterectomy indication } \\
\hline Myoma & $10(33.3)$ & $7(25.9)$ & \\
\hline Adenomyosis & $3(10.0)$ & $2(7.4)$ & \\
\hline Myoma and adenomyosis & $16(53.3)$ & $16(59.2)$ & \\
\hline Combined with cervical lesion & $1(3.4)$ & $2(7.5)$ & \\
\hline Surgical procedure & & & 0.812 \\
\hline Hysterectomy alone & $22(72.8)$ & $18(66.7)$ & \\
\hline Hysterectomy with adnexal surgery ${ }^{a}$ & $8(27.2)$ & $9(33.3)$ & \\
\hline
\end{tabular}

Data are presented as mean \pm standard deviation or number (\%).

TUM, transumbilical morcellation; TVM, transvaginal morcellation.

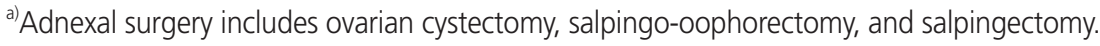




\section{Obstetrics \& Gynecology Science}

Seung Ju Oh, et al. Transumbilical \& transvaginal morcellation

\section{Results}

Between March 2013 and July 2017, 386 patients underwent SPA-TLH. In total, 329 patients were excluded from the study according to the exclusion criteria. Meanwhile, $57 \mathrm{pa}-$ tients were enrolled, and 30 and 27 patients underwent SPATLH with TUM and TVM, respectively, for tissue extraction.

The baseline characteristics, except for a history of cesarean section and vaginal delivery, were similar between the TUM and TVM groups (Table 1). In the TUM group, 25 patients $(83.3 \%)$ had a history of cesarean section. In the TVM group, four patients $(14.8 \%)$ had a history of cesarean section $(P=0.002)$. Moreover, only 2 patients $(6.7 \%)$ had a history of vaginal delivery in the TUM group, and 24 (88.8\%) had a history of vaginal delivery in the TVM group $(P=0.001)$. In the TUM group, 22 patients underwent hysterectomy alone, and 8 had hysterectomy with adnexal surgery. Moreover, in the TVM group, 18 patients underwent hysterectomy, and 9 had hysterectomy with adnexal surgery.

The perioperative outcomes between the two study groups are depicted in Table 2 . The mean total operative time was
$135.3 \mathrm{~min}$ in the TUM group and $129.4 \mathrm{~min}$ in the TVM group ( $P=0.724)$. The mean total operative time was shorter in the TVM group than in the TUM group. However, the result was not significantly different. The mean tissue extraction time was $25.3 \pm 12.5$ minutes in the TUM group and $29.3 \pm 11.5$ minutes in the TVM group ( $P=0.243)$. The mean tissue extraction time was shorter in the TUM group than in the TVM group. However, no significant difference was observed. The extension of umbilical skin incision was noted in both groups. In the TUM group, the umbilical incision length was extended from 2.5 to $2.8 \mathrm{~cm}$. Moreover, in the TVM group, the umbilical incision length was extended from 2.5 to $2.7 \mathrm{~cm}$. No statistically significant difference was observed in both preoperative and postoperative skin incision lengths ( $P=0.672, P=0.091$ ). In addition, the amount of blood loss, decrease in hemoglobin level, length of postoperative hospital stay (days), and postoperative pain between the two groups did not significantly differ.

The weight of the uterus was significantly different between the two study groups ( $P=0.042)$. The median uterine weight of the TUM group was $735 \mathrm{~g}$, ranging from 520 to

Table 2. Perioperative outcomes $(n=57)$

\begin{tabular}{|c|c|c|c|}
\hline & TUM $(n=30)$ & TVM $(n=27)$ & $P$-value \\
\hline Total operation time (min) & $135.3 \pm 32.4$ & $129.4 \pm 33.6$ & 0.724 \\
\hline Morcellation time (min) & $25.3 \pm 12.5$ & $29.3 \pm 11.5$ & 0.243 \\
\hline Estimated blood loss (mL) & $90(15-300)$ & $60(20-380)$ & 0.582 \\
\hline $\mathrm{Hb}$ decrease (mg/dL) & $1.5 \pm 1.1$ & $1.4 \pm 0.9$ & 0.593 \\
\hline Postoperative hospital (days) & $3(2-5)$ & $3(2-4)$ & 0.876 \\
\hline \multicolumn{4}{|l|}{ Postoperative pain } \\
\hline Immediately after surgery & $3.56 \pm 0.52$ & $3.74 \pm 0.42$ & 0.847 \\
\hline $12 \mathrm{hr}$ after surgery & $3.14 \pm 0.67$ & $3.11 \pm 0.78$ & 0.773 \\
\hline $24 \mathrm{hr}$ after surgery & $2.54 \pm 0.84$ & $2.56 \pm 0.75$ & 0.452 \\
\hline $48 \mathrm{hr}$ after surgery & $2.10 \pm 0.79$ & $2.13 \pm 0.58$ & 0.574 \\
\hline Uterine weight (g) & 735 (520-1380) & $622(514-975)$ & 0.042 \\
\hline \multicolumn{4}{|l|}{ Length of skin incision (cm) } \\
\hline Preoperative & $2.5(2.4-2.7)$ & $2.5(2.3-2.8)$ & 0.672 \\
\hline Postoperative & $2.8(2.6-3.2)$ & $2.7(2.4-3.0)$ & 0.091 \\
\hline \multicolumn{4}{|c|}{ Major postoperative complication } \\
\hline Ureter injury & 1 & 0 & \\
\hline Re-operation & 0 & 0 & \\
\hline Re-admission & 0 & 0 & \\
\hline
\end{tabular}

Data are presented as mean \pm standard deviation or median (range).

TUM, transumbilical morcellation; TVM, transvaginal morcellation; Hb, hemoglobin. 


\title{
Obstetrics \& Gynecology Science
}

\author{
Vol. 63, No. 3, 2020
}

$1,380 \mathrm{~g}$. The median uterine weight in the TVM group was $622 \mathrm{~g}$, ranging from 514 to $975 \mathrm{~g}$.

No surgery was converted to multi-port laparoscopy or laparotomy. In the TUM group, 1 patient presented with intraoperative ureter injury. Primary repair of the ureter and retrograde pyelography-guided ureter stent insertion were conducted. The ureter stent was removed 3 months after the operation, and no further treatment was required.

\section{Discussion}

No difference was observed in total operative time, tissue extraction time, or estimated amount of blood loss between the two study groups. Furthermore, postoperative decrease in hemoglobin level, length of hospital admission, and postoperative pain did not significantly differ. Uterine weight was significantly different between the two study groups (TUM: $735 \mathrm{~g}$ [520-1,380 g], TVM: $622 \mathrm{~g}$ [514-975 g]; $P=0.042)$. However, no significant difference was observed in tissue extraction time between TUM and TVM (25.3 vs. 29.3 minutes; $P=0.243$ ). Thus, a larger specimen can be retrieved using the TUM procedure within the same duration.

In total, 24 patients (88.8\%) in the TVM group and two patients $(6.7 \%)$ in the TUM group had a history of vaginal delivery $(P=0.001)$. The repeated stretching of vaginal tissues, such as that during vaginal delivery, leads to decreased collagenase in these tissues and enlargement of the vaginal cavity [13]. In patients with an enlarged vaginal cavity, TVM is a more competitive procedure. However, in patients with a history of cesarean section but without vaginal delivery, the vaginal cavity is relatively narrow, and TUM is more effective for specimen extraction.

According to a previous study, adhesiolysis of the posterior uterus, time to colpotomy completion, uterine extraction time, and uterine weight are the predictors of total operative time in performing SPA-TLH. Additionally, a positive correlation was observed between uterine weight and extraction time [6]. To reduce the overall operative time, the specimen extraction time must be decreased.

Several technical attempts have been made in removing a large uterus using procedures, including intra-abdominal morcellation with a power morcellator, mini-laparotomy, TVM, and TUM $[7,8,10]$. Intra-abdominal morcellation with a laparoscopic power morcellator was widely performed before the release of the November 2014 Food and Drug Administration safety communication statement about the risk of using morcellators for uterine fibroid morcellation due to the risk of specimen spillage and tumor dissemination [14]. In addition to the risk of specimen spillage, laparoscopic morcellation has been associated with bowel or vessel injury caused by the morcellator blade as well as prolonged operative time $[10,15]$.

Transvaginal specimen extraction is generally performed using bisection, morcellation, wedge resection, and vaginal myomectomy. However, this procedure is challenging to perform, and the total operative time is higher in patients with a narrow vaginal cavity and obesity [16]. During transvaginal tissue extraction, injury in the vaginal wall, vaginal stump, bladder, and ureter may occur [17].

Transumbilical tissue extraction was first performed to remove huge pelvic masses, including ovarian masses. Recently, transumbilical tissue extraction in laparoscopic hysterectomy was found to be safe and effective. In addition, as TUM is performed with the specimen in an endoscopic bag, the risk of bowel injury, vessel injury, and tumor dissemination is reduced [18]. Moreover, our study showed the feasibility of TUM, without complications and extension of operative time.

In 2008, Ghezzi et al. [9] have reported that the transumbilical approach was feasible for adnexal masses. Furthermore, in 2012, they showed that the use of the vaginal approach in colpotomy might be more effective for the removal of adnexal masses [19]. However, the results of these studies differ from those of the current study because the target was an adnexal mass. This type of mass can be easily resected via a small incision because it is rarely firm. However, the uterus with total hysterectomy is solid and greater in size on which the procedure was performed were different in nature. A solid and larger uterus is more difficult to remove via the vaginal cavity and particularly through a narrow vaginal cavity.

A recent study has compared TVM and TUM during multiport laparoscopic hysterectomy, and results showed no difference between the 2 morcellation methods. Between January 2010 and December 2014, patients with a uterus weighing $\geq 500 \mathrm{~g}$ were included in the study, and all manual morcellation procedures were performed in an endoscopic bag. The abdominal approach was used in $58.7 \%$ of the patients and the vaginal approach in $41.3 \%$. Results showed no gross spillage, rupture of the endoscopic bag, or other complica- 


\section{Obstetrics \& Gynecology Science}

Seung Ju Oh, et al. Transumbilical \& transvaginal morcellation

tions correlated to the morcellation procedure [18].

Our study performed single-port-access laparoscopy, and no difference was observed between the TUM and TVM groups in terms of extension of the skin incision. Previous studies have shown that postoperative pain after laparoscopy significantly decreases with the reduction in incision size [20]. Therefore, in this study, no difference was observed in postoperative pain between the two groups because the umbilical incision sizes were similar. In addition to reducing postoperative pain, smaller umbilical incision sizes are beneficial in terms of cosmetic aspects.

An objective guideline for selecting morcellation method is not available. Thus, this might have caused selection bias as the selection of procedure was not blinded or randomized. Moreover, since this was a retrospective study with a small sample size, a randomized prospective analysis should be performed to increase the power of the study. In this study, all patients were Koreans, and they had a relatively low BMI. Therefore, there is a limitation for the generalization and application of the results. In addition, there might be errors in the measurement of skin incisions. To evaluate the exact extension, the length of the incision in the fascia before and after the operation must be compared. The stability of the tissue extraction method and the use of an endo-pouch has not been well established. Thus, further prospective studies must be conducted.

Future large-scale prospective randomized controlled trials about specimen extraction methods may provide more reliable results and objective guidelines for selecting the morcellation method.

In conclusion, when performing SPA-TLH in a patient with a large uterus, TUM is considered safe and effective. In particular, in patients with a narrow vaginal cavity, TUM may be a more suitable procedure for specimen retrieval.

\section{Acknowledgements}

This work was supported by the Yonsei University Wonju Campus Future-Leading Research Initiative of 2018 (201862-0040) and by the Basic Science Research Program through the National Research Foundation of Korea (NRF) funded by the Ministry of Education, Science and Technology (NRF-2017R1D1A3B03032983).

\section{Conflict of interest}

No potential conflict of interest relevant to this article was reported.

\section{Ethical approval}

The study was approved by the Institutional Review Board of Wonju Severance Christian Hospital (IRB No. CR318116) and performed in accordance with the principles of the Declaration of Helsinki. Written informed consents were obtained.

\section{Patient consent}

The patients provided written informed consent for the publication and the use of their images.

\section{References}

1. Reich H, DeCarpio J, McGlynn F. Laparoscopic hysterectomy. J Gynecol Surg 1989;5:213-6.

2. Wu MP, Lee $C L$. The trends of minimally invasive surgery for benign gynecologic lesions, 1997-2007 in Taiwan. Gynecol Minim Invasive Ther. 2012;1:3-8.

3. Choi YS, Park JN, Oh YS, Sin KS, Choi J, Eun DS. Singleport vs. conventional multi-port access laparoscopyassisted vaginal hysterectomy: comparison of surgical outcomes and complications. Eur J Obstet Gynecol Reprod Biol 2013;169:366-9.

4. Pelosi MA, Pelosi MA 3rd. Laparoscopic hysterectomy with bilateral salpingo-oophorectomy using a single umbilical puncture. N J Med 1991;88:721-6.

5. Jackson T, Einarsson J. Single-port gynecologic surgery. Rev Obstet Gynecol 2010;3:133-9.

6. Jeung IC, Lee YS, Song MJ, Park EK. Laparoendoscopic single-site total laparoscopic hysterectomy: clinical factors that affect operative times and techniques to overcome difficulties. J Minim Invasive Gynecol 2017;24:617-25.

7. O'Shea RT, Cook JR, Seman El. Total laparoscopic hysterectomy: a new option for removal of the large myomatous uterus. Aust N Z J Obstet Gynaecol 2002;42:282-4.

8. Chen SY, Chang DY, Sheu BC, Torng PL, Huang SC, Hsu 


\section{Obstetrics \& Gynecology Science}

Vol. 63, No. 3, 2020

WC, et al. Laparoscopic-assisted vaginal hysterectomy with in situ morcellation for large uteri. J Minim Invasive Gynecol 2008;15:559-65.

9. Ghezzi F, Cromi A, Uccella S, Siesto G, Bergamini V, Bolis P. Transumbilical surgical specimen retrieval: a viable refinement of laparoscopic surgery for pelvic masses. BJOG 2008;115:1316-20.

10. Serur E, Lakhi N. Laparoscopic hysterectomy with manual morcellation of the uterus: an original technique that permits the safe and quick removal of a large uterus. Am J Obstet Gynecol 2011;204:566.e1-2.

11. Kovac SR. Route of hysterectomy: an evidence-based approach. Clin Obstet Gynecol 2014;57:58-71.

12. Hawker GA, Mian S, Kendzerska T, French M. Measures of adult pain: Visual Analog Scale for Pain (VAS Pain), Numeric Rating Scale for Pain (NRS Pain), McGill Pain Questionnaire (MPQ), Short-Form McGill Pain Questionnaire (SF-MPQ), Chronic Pain Grade Scale (CPGS), Short Form-36 Bodily Pain Scale (SF-36 BPS), and Measure of Intermittent and Constant Osteoarthritis Pain (ICOAP). Arthritis Care Res (Hoboken) 2011;63 Suppl 11:S240-52.

13. Zong W, Jallah ZC, Stein SE, Abramowitch SD, Moalli PA. Repetitive mechanical stretch increases extracellular collagenase activity in vaginal fibroblasts. Female Pelvic Med Reconstr Surg 2010;16:257-62.

14. U.S. Food and Drug Administration. Recommended labeling statements [Internet]. Silver Spring (MD): Food and Drug Administration; 2014 [cited 2014 Nov 24]. Available from: https://www.fda.gov/downloads/medicaldevices/safety/alertsandnotices/ucm424444.pdf.

15. Kho RM. In the aftermath of the storm called power morcellation. J Minim Invasive Gynecol 2016;23:847-8.

16. Wu KY, Lertvikool S, Huang KG, Su H, Yen CF, Lee CL. Laparoscopic hysterectomies for large uteri. Taiwan J Obstet Gynecol 2011;50:411-4.

17. Wang H, Li P, Li X, Gao L, Lu C, Zhao J, et al. Total laparoscopic hysterectomy in patients with large uteri: comparison of uterine removal by transvaginal and uterine morcellation approaches. BioMed Res Int 2016;2016:8784601.

18. Serur E, Zambrano N, Brown K, Clemetson E, Lakhi N. Extracorporeal manual morcellation of very large uteri within an enclosed endoscopic bag: our 5-year experience. J Minim Invasive Gynecol 2016;23:903-8.

19. Ghezzi F, Cromi A, Uccella S, Bogani G, Serati M, Bolis P. Transumbilical versus transvaginal retrieval of surgical specimens at laparoscopy: a randomized trial. Am J Obstet Gynecol 2012;207:112.e1-6.

20. Acton JN, Salfinger SG, Tan J, Cohen PA. Outcomes of total laparoscopic hysterectomy using a $5-\mathrm{mm}$ versus 10-mm laparoscope: a randomized control trial. J Minim Invasive Gynecol 2016;23:101-6. 